\title{
A Clustering Based Method to Stipulate the Number of Hidden Neurons of mlp Neural Networks: Applications in Pattern Recognition
}

M.R. SILVESTRE, S.M. OIKAWA 2 , Departamento de Matemática, Estatística e Ciência da Computação, FCT, UNESP, 19060-900 Presidente Prudente, SP, Brasil.

F.H.T. VIEIRA, Engenharia Elétrica e de Computação, UFG, 74605-010 Goiânia, GO, Brasil.

L.L. LING, ${ }^{4}$ Departamento de Comunicações, FEEC, UNICAMP, 13083-970 Campinas, SP, Brasil.

Abstract. In this paper, we propose an algorithm to obtain the number of necessary hidden neurons of single-hidden-layer feed forward networks (SLFNs) for different pattern recognition application tasks. Our approach is based on clustering analysis of the data in each class. We show by simulations that the proposed approach requires less computation $\mathrm{CPU}$ time and error rates as well as a smaller number of neurons than other methods.

Key-words. Hidden neurons, SLFN neural network, cluster analysis.

\section{Introduction}

In [3], the authors demonstrated that standard single-hidden-layer feedforward networks (SLFNs) possessing $\mathrm{N}$ hidden neurons and bounded nonlinear activation hidden function, can recognize $\mathrm{N}$ distinct patterns with zero error. These authors also deduced an upper bound on the number of hidden neurons that are valid only for linear activation output function. Moreover, they claim that the neural network construct by their method may have redundancy in some applications, due to 1) in trying to obtain zero-error precision and 2) the correlation between the activation function and the given samples. Theses two aspects give rise to the problem of optimum network construction. In most applications, the error can be larger than zero and the number of hidden neurons can be less than the upper bound.

MLP (Multi Layered Perceptron) neural networks with sigmoid activation functions provide hyperplanes that divide the input space in classification regions [5].

\footnotetext{
${ }^{1}$ miriam@fct.unesp.br

2 oikawa@fct.unesp.br

${ }^{3}$ flavio@eee.ufg.br

${ }^{4}$ lee@decom.fee.unicamp.br
} 
Therefore, one can construct hyperplanes by using the weights of the network. We present an example of such a construction considering the XOR problem in Section 2.1 .

Clustering techniques allow us to aggregate patterns that are close in space, based on distance metrics. In this sense, clusters are used to divide the input patterns in similar groups that can occupy different positions in the input space.

If there are two different neighbor clusters, it is possible to imagine that there is a hyperplane that can separate these clusters. Then, if the number of cluster of a dataset is known, we can assume that the same number (or less) of hyperplanes are dividing them.

The initialization of neural networks through clustering techniques is the main subject of many researches [2] and [9]. In [2], it is proposed an algorithm to initialize and optimize the MLP neural networks that does a particularly different pre-processing in all input data and after that uses cluster analysis in step 2 and 3 to define weights of the hidden layer. Further, they propose an optimization of the neural network based on penalty term. In the article [9], the authors suggest the use of clustering and optimization techniques to select the hidden units of the initial neural network configuration, but their method need to optimize a lot of different sizes of network.

In this work, we propose an algorithm to define an appropriate number of hidden neurons of a MLP neural network, based on clustering methods. Our method is simple than [2], because the variables are transformed to have zero mean and one variance, and we used the standard backpropagation algorithm, without optimization of penalty parameters such as used by [2]. Also, we do not use clusters optimization to select the size of the network as suggested by [9]. We investigated the results obtained by our method using $\mathrm{N}$ hidden neurons sigmoid activation functions to output layer, instead of the linear activation output function proposed in [3], because they are more appropriated to pattern recognition and classification problems.

The rest of this paper is organized as follows. In Section 2, we recall some basic theory involving neural networks and clustering techniques. In Section 3 we present a description of the proposed cluster analysis method. In Section 4, we evaluate the efficiency of the proposed algorithm by simulations and we discuss the obtained results. Finally, in Section 5, we conclude.

\section{MLP Neural Network and Cluster Analysis}

In this section, first of all we present some definitions related to the MLP neural network in Section 2.1. Next, in Section 2.2, we give a general overview of cluster analysis.

\subsection{MLP Network}

Consider a MLP network, with one hidden layer (SLFN). Let $[\mathbf{x}(n), \mathbf{t}(n)]$ denote the $n^{\text {th }}(n=1, \ldots, N)$ training pattern where $\mathbf{x}(n)=\left[\mathbf{x}_{1}(n), \ldots, \mathbf{x}_{d}(n)\right]^{T}$ is the $d$ - 
dimensional input feature vector and $\mathbf{t}(n)=\left[t_{1}(n), \ldots, t_{c}(n)\right]^{T}$ corresponds to the desired $c$-class output response. Let $\mathbf{W}^{(1)}=\left\{w_{j i}^{(1)}(n)\right\}$ and $\mathbf{W}^{(2)}=\left\{w_{k j}^{(2)}(n)\right\}$ be the matrix of synaptic weights which connect the input to hidden layers and the hidden to the output layers, respectively. The optimization procedure for classification consists of minimizing the sum of the mean square errors between the network output $y_{k}(n)$ and the desired output $t_{k}(n)$, i.e.:

$$
E=\sum_{n=1}^{N} E(n), \text { where } E(n)=\frac{1}{2} \sum_{k=1}^{c}\left[y_{k}(n)-t_{k}(n)\right]^{2} .
$$

Assuming that $g^{(1)}$ and $g^{(2)}$ are activation functions of sigmoid logistic type (equation $(2.1))$ :

$$
g^{(\cdot)}\left(v_{j}(n)\right)=\frac{1}{1+\exp \left(-p v_{j}(n)\right)}
$$

the $k^{t h}$ network output $y_{k}(n)$ is given by $y_{k}(n)=g^{(2)}\left(\sum_{j=0}^{M} w_{k j}^{(2)}(n) g^{(1)}\left(v_{j}(n)\right)\right)$, where $M$ is the number of hidden neurons. If the equation (2.1) is applied to the hidden layer, then $v_{j}(n)=\sum_{i=0}^{d} w_{j i}^{(1)}(n) x_{i}(n)$; but if it is applied to the output layer, $v_{j}(n)=\sum_{j=0}^{M} w_{k j}^{(2)}(n) g^{(1)}\left(\sum_{i=0}^{d} w_{j i}^{(1)}(n) x_{i}(n)\right)$. Notice that the number of hidden neurons in the hidden layer defines the maximum number of hyperplanes that delimit decision boundaries in input feature space.

To exemplify how the delimit decision boundaries are created by the hidden neurons, we will consider the XOR problem (Exclusive OR) with $N=4$ patterns, $p=2$ variables $\left(x_{1}\right.$ and $\left.x_{2}\right)$ and $c=2$ classes $(0$ and 1$)$. The input patterns are: $(0,0)$ and $(1,1)$ that produces the class 0 ; and $(0,1),(1,0)$ belong to class 1 .

The training of a SLFN with $M=4$ hidden neurons produces the $\mathbf{W}^{(1)}$ matrix. To each row (neuron) $j=1, \ldots, 4$ of $\mathbf{W}^{(1)}$ matrix, it is possible to construct a hyperplane by varying the $x_{1}$ and $x_{2}$ variables according to the equation:

$$
w_{j 1}^{(1)} x_{1}+w_{j 2}^{(1)} x_{2}+w_{j 0}^{(1)}(-1)=0 .
$$

The resulting $M=4$ hyperplanes are presented in Figure 1 (graphic on the left), and a more careful analysis of this Figure reveals that there are redundancies, e. g. the problem need less than $M=4$ hyperplanes to discriminate the two classes.

A SLFN with $M=2$ hidden neurons can solve the XOR problem. The resulting $M=2$ hyperplanes are presented in Figure 1 (graphic on the right). It is clear that the hyperplanes built with the weights of the hidden layer are able to divide the input feature space in regions.

\subsection{Cluster analysis}

In this section, we will focus on the clusters analysis. Traditional hierarchical clustering algorithms can be applied in classification tasks of many types of datasets. The main purpose in cluster analysis is to aggregate similar patterns in groups. 

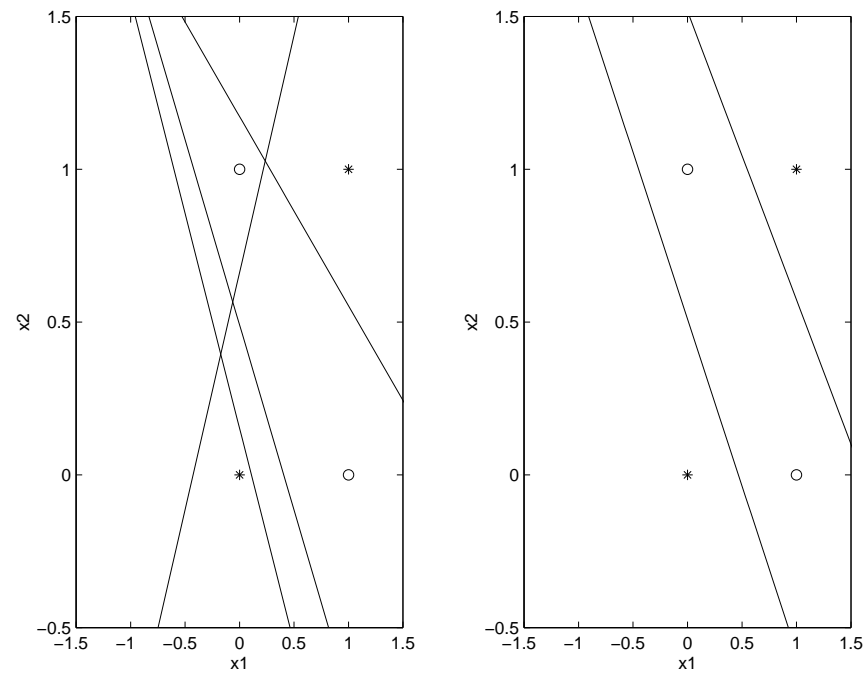

Figure 1: The XOR problem resolved with $M=4$ hyperplanes (graphic on the left); and with $M=2$ hyperplanes (graphic on the right).

Firstly, it is necessary to define which measure of similarity or dissimilarity is more appropriate for the clustering procedure. This measure is called 'resemblance coefficient' and it is computed to each pair of patterns. An example of resemblance coefficient is the Euclidean distance (equation (2.2)):

$$
d_{a b}=\sqrt{\left(x_{1}-y_{1}\right)^{2}+\left(x_{2}-y_{2}\right)^{2}+\ldots+\left(x_{d}-y_{d}\right)^{2}} .
$$

There are others distance measures as Mean Euclidean distance, City-Block or Manhattan distance, Minkowsky distance, coefficients of Gower, Catell, Camberra, Bray-Curtis, Sokal-Sneath, Cosine, Pearson Correlation and many others.

Second, which method of clustering is to be used, the hierarchical clustering techniques proceed by either a series of successive mergers, called Agglomerative hierarchical methods, or a series of successive divisions, called Divisive hierarchical methods. Agglomerative hierarchical methods start with the individual objects. These methods initiate the algorithm with all patterns and they are aggregated until the last one.

In this work, we pay attention on some methods classified as Agglomerative hierarchical methods such as: Single Linkage, Complete Linkage, Average Linkage, Median Linkage, Centroid, Ward and others.

In [4], the authors present an Agglomerative hierarchical method for clustering $N$ objects (in our case these objects are the patterns):

1. Start with $N$ clusters, each containing a single entity and an $N \times N$ symmetric matrix of distances (or similarities) $\mathbf{D}=\left\{d_{a b}\right\}$.

2. Search the distance matrix for the nearest (most similar) pair of clusters. Let the distance between "most similar" clusters $U$ and $V$ be $d_{U V}$. 
3. $\quad$ Merge clusters $U$ and $V$. Label the newly formed cluster $(U V)$. Update the entries in the distance matrix by (a) deleting the rows and columns corresponding to clusters $U$ and $V$ and (b) adding a row and column giving the distances between cluster $(U V)$ and the remaining clusters.

4. Repeat Steps 2 and 3 a total of $N-1$ times. (All objects will be in a single cluster after the algorithm terminates). Record the identity of clusters that are merged and the levels (distances or similarities) at which the mergers take place.

Now, we will describe some aspects and procedures belonging to the Agglomerative clustering methods. Initially one has to find the smallest distance in $\mathbf{D}=\left\{d_{a b}\right\}$ and merge the corresponding patterns, say, $U$ and $V$, to get the cluster $(U V)$. For Step 3 of the general algorithm the distances between $(U V)$ and any other cluster $W$ are computed by different ways in each clustering method: Single Linkage, Complete Linkage, Average Linkage, Ward, Median Linkage and Centroid (more details can be seen in [4]). Then, after the clustering method be applied there was construct a new distance symetric matrix $\mathbf{D}_{c}=\left\{d_{a b}\right\}$.

After the application of one or more methods mentioned above, it is necessary to define how many clusters there are on the data, because the Agglomerative algorithm aggregate all the patterns until just one cluster. Then it is necessary to found a cut point on the dendrogram or tree graphic. The dendrogram is built with the measure of distance in each pass of the clustering.

To define how is the better point to cut the tree you have to find a point where the clustering of one pass to the next pass provided a large increase in the distance or a jump. This means that were jointed two patterns/clusters that do not have much in common, so a large distance measure was produced between them. So, it is convenient decide to the number of clusters provided by the pass anterior to the jump. The jump can be easily seen if we construct a graph with the distance levels in each step of the linkage method. In this case, the number of clusters before this jump was more adequate to the data. Figure 2 illustrates the behavior of the distance analysis (D) to class 2 of glass training dataset given in Section 4 . The largest jump in distance is at penultimate step. Then, the optimum number of clusters to this class is two. This method is further being called distance analysis (D) throughout this paper.

To define the number of clusters it is possible to use many other measures such as: the sum of squares between the groups $\left(R^{2}\right)$; semi-partial correlation $\left(S P R^{2}\right)$; pseudo F statistic, pseudo $T^{2}$ statistic and cubic clustering criterion $(C C C)$.

In each step of the clustering algorithm, the total sum of squares $\left(T_{c}\right)$, between $(B)$ and within $(W)$ the clusters constructed are calculated. Then, the $R^{2}$ coefficient is defined as $R^{2}=\frac{B}{T_{c}}$. More details of these other measures can be seen in [6]. The maximum value of $R^{2}$, is one and it is obtained when the number of groups is the same as the number of patterns. Our experiments indicated that values of $R^{2} \geq 0.80$ are considered to provide a reasonable number of clusters.

The final concern of is to decide what is the most adequate clustering method for a given dataset. In [7], the author suggests the cophenetic correlation coefficient to decide the better clustering method to apply to the input data. The cophenetic correlation coefficient measures how similar are the tree, represented by the distance symetric matrix $\mathbf{D}_{c}=\left\{d_{a b}\right\}$ and the initial resemblance matrix $\mathbf{D}=\left\{d_{a b}\right\}$. The 


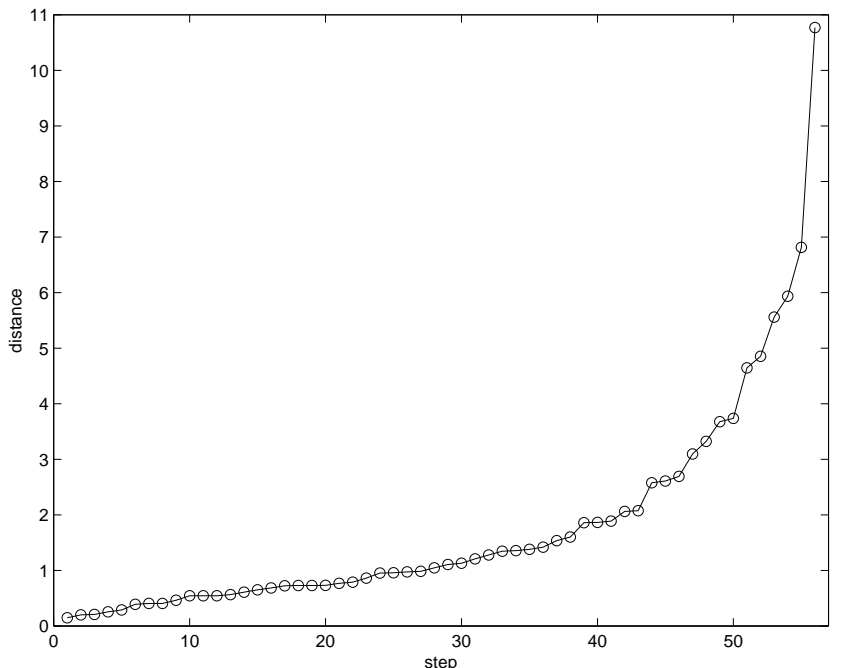

Figure 2: Behavior of the distance analysis (D) to Glass dataset (class 2).

cophenetic correlation is just a measure of correlation between all pairs of elements of this two symetric distance matrix. Values of cophenetic correlation near 1.0 represent a good concordance/match, but in practice the relationship is not exact. Then, values of cophenetic correlation of 0.8 or above indicates that the dendrogram does not greatly distort the original structure in the input data ([7], p.27).

In the next section, we present an algorithm to define the number of initial hidden neurons of a SLFN using cluster analysis.

\section{Cluster analysis based method}

Our proposal consists of firstly applying the hierarchical clustering algorithms in each class $(k=1,2, \ldots, c)$ of the considered problem and to use some method to define the number of clusters in the classes. We fix the number of hidden neurons $M$ of a SLFN as being equal to the sum of all number of clusters for all classes.

The proposed algorithm is outlined below.

Consider a classification problem containing $c$ classes and $d$ input variables. Then, perform the following steps:

1. Let $k=1$. Use just the data of the $k^{t h}$ class.

2. Apply various cluster algorithms to the data.

3. Calcule the cophenetic correlation to each cluster method and choose that method with the highest correlation.

4. To the cluster method defined in step 3, find the number of clusters in data using some measures such as the distance analysis D and $R^{2} \geq 0.8$ (see Section 2.2).

5. Set the number of clusters given in step 4 as being $g_{k}$.

6 . Let $k=k+1$. 
7. Repeat the steps 2 until 6 for all classes or until $k=c$.

8. Define the initial number of hidden neurons of a SLFN as being $M=\sum_{k=1}^{c} g_{k}$.

\section{Results and Discussion}

In our experiments we considered a SLFN with $d$ input neurons, the same number as the variables of the problem in study; $c$ output neurons, exactly the number of classes of the problem. The training networks were did using the backpropagation algorithm. And to find the $M$ hidden neurons it were used the following methods:

1. Our algorithm with $M$ defined by distances analysis (D);

2. Our algorithm with $M$ defined by $R^{2} \geq 0.8$;

3. Stem-and-leaf graphic technique proposed by [8];

4. The number $N$ of training pattern discussed in [3], but we applied a sigmoid logistic function to output layer.

In [8] is presented the stem-and-leaf graphic technique, that is constructed using a stem-and-leaf graphic of the first principal component obtained to the training data. The number of occupied stems is counted and this number is set as the number of hidden neurons to one class. In summary, this method is applied to each class separately and the occupied stems are added to produce the final number of hidden neurons.

\subsection{Experiment 1: Glass Dataset}

We analyzed the Glass classification problem, available in [1]. The study of classification of glass types was motivated by criminological investigation. At the scene of the crime, the glass left can be used as evidence if it is correctly identified [1]. The dataset for the glass problem has 214 patterns with $d=9$ variables, $c=6$ classes or type of glass: class 1 (building windows float processed), class 2 (building windows non float processed), class 3 (vehicle windows float processed), class 4 (containers), class 5 (tableware), class 6 (headlamps). We divided this dataset in two parts: training $\left(N_{\text {train }}=161\right.$ or $\left.75 \%\right)$ and testing dataset $\left(N_{\text {test }}=53\right.$ or $\left.25 \%\right)$, taking into account the percents of each class.

The description of the Glass dataset given in [1] indicates that all input variables are of real type. First, we compute the means and variances of all training dataset variables. These results were used to transform the test dataset with the same parameters of the training dataset. Next, all variables were transformed to have zero mean and one variance.

In this work we considered the Euclidean distance (equation 2.2) as a resemblance coefficient. We calculated the cophenetic correlation coefficients separately to each class. The Table 1 shows the results to the glass problem.

The bold numbers in Table 1 represent the maximum cophenetic correlation to each class. Then, the behavior of the distances analysis (D) must be made with the method indicated with the bold numbers. Notice that all cophenetic correlations are greater than 0.8 , indicating good match. In this problem we need to apply the Centroid Linkage method at classes 1 and 3 and Average Linkage at classes 2, 4, 5 
Table 1: Cophenetic correlation of clustering methods applied to Glass training dataset.

\begin{tabular}{|c|c|c|c|c|c|c|}
\hline Class & Simple & Average & Centroid & Complete & Median & Ward \\
\hline 1 & 0.9073 & 0.8850 & 0.9227 & 0.8683 & 0.8149 & 0.8416 \\
\hline 2 & 0.9503 & 0.9742 & 0.9511 & 0.9572 & 0.9442 & 0.8916 \\
\hline 3 & 0.9080 & 0.9237 & 0.9275 & 0.9194 & 0.9194 & 0.9003 \\
\hline 4 & 0.9782 & 0.9855 & 0.9663 & 0.9827 & 0.9543 & 0.9654 \\
\hline 5 & 0.9140 & 0.9275 & 0.9190 & 0.9231 & 0.9182 & 0.8535 \\
\hline 6 & 0.9573 & 0.9773 & 0.9622 & 0.9341 & 0.9065 & 0.7992 \\
\hline
\end{tabular}

and 6 . The numbers of clusters indicated for each class are: 5, 2, 2, 3, 2 and 2 . We obtained a total number of clusters equals to 16 . Thus, we adopted $M=16$ hidden neurons to the method D.

In this problem, we chose the sigmoid as activation function, equation (2.1), with $p=0.8$ in the hidden and output layers. We used the backpropagation algorithm to adjust the weights. The following configuration set is considered: a learning rateparameter equals to 0.5 , momentum term $=0$ and stopping criterion dew $\leq 0.05$ (it is considered to have converged when the Euclidean norm of the gradient vector reaches a sufficiently small gradient threshold).

Table 2 presents the results obtained with different sizes of $M$ (2nd to 5 th columns), according to the four methods described in the beginning of the Section 4. We executed 100 runs of the MLP network, with each configuration of hidden number neurons (2nd row), and calculated: the mean epochs spent to converge (3rd row), CPU time spent with the training and test (4th row), the mean training error rate (Etrain) in perceptual and standard deviation in brackets (5th row) and the results to Etest (6th row). The 7th and 8 th rows are the First and the Third Quartiles Etest, these statistics represents $25 \%$ and the $75 \%$ smallers and results, respectively, and given an idea of the spread of the values of Etest. The last row indicates the best network test error rate obtained by choosing the smaller training error rate produced on all 100 runs, each one with different random initial hidden and output weights.

It can be observed in Table 2 that the methods $R^{2} \geq 0.80$ and RF produced smaller test error average rates (Mean Etest) than the other two methods; and the first and the third quartiles were also smaller. The method D requires too much training epochs, but it is the fastest method with CPU time at about 2:25 hours, using a computer with an Intel Celeron M360 Processor and a 256 Mbytes of DDR RAM Memory, and provided the best network test error rate (24.528).

\subsection{Experiment 2: Ecoli Dataset}

This dataset, found in [1], is related to protein localization sites, and contains 336 patterns with $d=7$ variables, $c=8$ classes or type of glass: class 1 (cytoplasm), class 2 (inner membrane without signal sequence), class 3 (perisplasm), class 4 (inner membrane, uncleavable signal sequence), class 5 (outer membrane), class 6 (outer membrane lipoprotein), class 7 (inner membrane lipoprotein) and class 8 (inner 
Table 2: On Glass dataset for 100 runs of different methods to define the number of hidden neurons.

\begin{tabular}{|c|c|c|c|c|}
\hline Class & $\mathrm{D}$ & $R^{2} \geq 0.80$ & $\mathrm{RF}$ & Ntrain \\
\hline Hidden Neurons & 16 & 39 & 45 & 162 \\
\hline Mean Epoch & 190.96 & 128.99 & 105.88 & 42.06 \\
\hline CPU time (h:min:sec) & $2: 25: 34$ & $3: 48: 01$ & $3: 38: 03$ & $4: 59: 38$ \\
\hline Mean Etrain (\%) (Std. Dev.) & $9.248_{(2.244)}$ & $14.863(3.098)$ & $17.919(3.407)$ & $32.559(5.990)$ \\
\hline Mean Etest (\%) (Std. Dev.) & $32.321(4.037)$ & $31.075(1.905)$ & $30.943(1.989)$ & $35.132(4.735)$ \\
\hline First Quartile Etest & 30.189 & 30.189 & 30.189 & 32.075 \\
\hline Third Quartile Etest & 35.849 & 32.075 & 32.075 & 37.736 \\
\hline Best Net Etest & 24.528 & 32.075 & 32.075 & 32.075 \\
\hline
\end{tabular}

Table 3: Cophenetic correlation of clustering methods applied to Ecoli training dataset.

\begin{tabular}{|c|c|c|c|c|c|c|}
\hline Class & Simple & Average & Centroid & Complete & Median & Ward \\
\hline 1 & 0.6205 & 0.6876 & 0.6267 & 0.4372 & 0.6291 & 0.4217 \\
\hline 2 & 0.6714 & 0.7720 & 0.7438 & 0.4990 & 0.6380 & 0.4403 \\
\hline 3 & 0.8268 & 0.8683 & 0.8458 & 0.7805 & 0.8100 & 0.5293 \\
\hline 4 & 0.6276 & 0.6578 & 0.6484 & 0.5818 & 0.5860 & 0.4789 \\
\hline 5 & 0.6747 & 0.7200 & 0.5197 & 0.5550 & 0.5753 & 0.6283 \\
\hline 6 & 0.9777 & 0.9978 & 0.9768 & 0.9770 & 0.9752 & 0.9778 \\
\hline
\end{tabular}

membrane cleavable signal sequence). The dataset was divided in two parts: $75 \%$ to training (Ntrain) and $25 \%$, to testing dataset (Ntest).

We applied the transformation of zero mean and one variance in the training and test datasets. Besides, we separately calculated the cophenetic correlation coefficients to each class. Table 3 depicts the results to the Ecoli problem.

The bold numbers in Table 3 represent the maximum cophenetic correlation to each class. Then, the behavior of the distances analysis (D) must be made with the method indicated with the bold numbers. For example, in this problem we need to apply Average Linkage at all classes. Notice that just for classes 3 and 6 the cophenetic correlation were greater than $0.8,0.9978$ and 0.8683 , respectively. To the other classes, the cophenetic correlation are not so high, and resulted between 0.6578 and 0.7720 . The numbers of clusters indicated for each class were: $7,2,3,7$, 14, 2, 1 and 1. To the classes 7 and 8 it was impossible to calculate the cophenetic correlation because they have just one pattern in each class to training dataset, then to this class it was adopted just 1 cluster for each them. In this way, the total clusters number was 37 .

After that, we executed 100 runs of MLP network, with each random configuration of hidden number neurons. Table 4 presents the results obtained with different sizes of M, according to the four methods described in the beginning of the Section 4. To this problem we used the same estructure of training as used to Glass, except to stop criterium that was defined to 500 training epochs. It was did because in the Ecoli data the other stop criterium $($ dew $\leq 0.05)$ was finishing the training with many few epochs (at about 28 epochs), and the results were not good. 
Table 4: On Ecoli dataset for 100 runs of different methods to define the number of hidden neurons.

\begin{tabular}{|c|c|c|c|c|}
\hline Information & $\mathrm{D}$ & $R^{2} \geq 0.80$ & $\mathrm{RF}$ & Ntrain \\
\hline Hidden Neurons & 37 & 70 & 57 & 252 \\
\hline Mean Epochs & 500 & 500 & 500 & 500 \\
\hline CPU time (h:min:sec) & $11: 17: 24$ & $20: 33: 07$ & $16: 50: 05$ & $58: 11: 15$ \\
\hline Mean Etrain (\%)(Std. Dev.) & $7.1746(0.7727)$ & $8.4246(0.6741)$ & $7.9881_{(0.6877)}$ & $8.5995(0.3946)$ \\
\hline Mean Etest (\%)(Std. Dev.) & $20.071(1.532)$ & $18.964(1.377)$ & $19.690(1.420)$ & $19.454(1.230)$ \\
\hline First Quartile Etest & 19.048 & 17.787 & 19.048 & 19.048 \\
\hline Third Quartile Etest & 21.429 & 20.238 & 20.238 & 20.238 \\
\hline Best Net Etest & 16.667 & 16.667 & 20.238 & 22.6190 \\
\hline
\end{tabular}

Table 4 reveals that the proposed method together with $\mathrm{D}$ and $R^{2} \geq 0.80$ dendrogram cut points, produced good results principally in relation to mean error test (Mean Etest). We can note that the Etest first quartile to $R^{2} \geq 0.80$ is the lowerst value (17.787). The others two methods (RF and Ntrain) presented the Etest a little more high, near about each other (19.690 and 19.454). The CPU time spent by $\mathrm{D}, R^{2} \geq 0.80$ and $\mathrm{RF}$ were at about 11 to 20 hours, but the CPU time exploded to Ntrain (58:11 hours). In our simulations, the $R^{2} \geq 0.80$ method has a slightly smaller error rate than the RF, but spending more CPU time. We also verified that the $\mathrm{D}$ method was the fastest method but it produces a little high test error rate (20.071). Concerning the Best Net Etest, the D and $R^{2} \geq 0.80$ methods produced small errors (16.667).

\section{Conclusion}

In this work, we propose a clustering based method to state the number of hidden neurons that can produce good classification error rate. Our cluster based method uses much less CPU time consuming than [3] method, modificated with a mlp network with sigmoid activation output function.

We observed that the performance of the proposed method is comparable to other methods when applied to the glass problem. In this problem, we obtained high values of cophenetic correlation (approximately 0.9). This fact indicates that an efficient clustering of the input data is made. Therefore, the accurate cluster analysis enhanced the results of the proposed method. To the Ecoli problem, the cophenetic correlation is just high for class 3 and 6 , to other classes these values were not so high values, indicating that there are not so good concordance/match between the real cluster configuration and the configuration obtained by clustering methods. Probably, because this, we had to training longer than glass problem. But, it was obtained good classifications results.

Finally, we can say that the cluster analysis applied to the classes can produce a number of hidden neurons able to produce accurate neural networks.

Resumo. Neste artigo, propomos um algoritmo para obter o número necessário de neurônios intermediários de uma rede neural contendo uma única camada escondida 
para aplicações em diferentes tarefas de reconhecimento de padrões. O método é baseado na análise de agrupamentos dos dados de cada classe. É mostrado por simulação que o método proposto requer menor tempo computacional e apresenta menor razão de erro bem como um número menor de neurônios que outros métodos.

\section{References}

[1] A. Asuncion, D.J. Newman, "UCI Machine Learning Repository", Irvine, CA: University of California, School of Information and Computer Science, 2007. Available: http://www.ics.uci.edu/ mlearn/MLRepository.html

[2] W. Duch, R. Adamczak, N. Jankowski, Initialization and optimization of multilayered perceptrons, in "Proceedings of the 3th Conference on Neural Networks and Their Applications", pp. 105-110, 1997.

[3] G.B. Huang, H.A. Babri, Upper bounds on the number of hidden neuron in feedforward networks with arbitrary bounded nonlinear activation functions, IEEE Transactions on Neural Networks, 9, No. 1 (1998), 224-229.

[4] R.A. Johnson, D.W. Wichern, "Applied Multivariate Statistical Analysis", 5th ed., Prentice Hall, Upper Saddle River, 2002.

[5] R.P. Lippmann, Pattern classification using neural networks, IEEE Communications Magazine, (1989), 47-64.

[6] S.A. Mingoti, "Análise de Dados através de Métodos de Estatística Multivariada: uma Abordagem Aplicada", UFMG, Belo Horizonte, 2005.

[7] H.C. Romesburg, "Cluster Analysis for Researchers", Robert E. Krieger, Malabar, 1990.

[8] M.R. Silvestre, L.L. Ling, Optimization of neural classifiers based on Bayesian decision boundaries and idle neurons pruning, in "Proceedings of the 16th International Conference on Pattern Recognition", pp. 387-390, 2002.

[9] N. Weymaere, J.P. Martens, On the initialization and optimization of multilayer perceptrons, IEEE Transactions on Neural Networks, 5, No. 5, (1994), 738-751. 